\title{
Nanofilm versus Bulk Polymorphism in Wurtzite Materials
}

\author{
Ilker Demiroglu ${ }^{1}$ and Stefan T. Bromley ${ }^{1,2, *}$ \\ ${ }^{1}$ Departament de Química Física and Institut de Química Teòrica i Computacional, \\ Universitat de Barcelona, E-08028 Barcelona, Spain \\ ${ }^{2}$ Institució Catalana de Recerca i Estudis Avançats (ICREA), 08010 Barcelona, Spain
}

(Received 16 November 2012; published 13 June 2013)

\begin{abstract}
We generate a wide range of hexagonal sheet-based $\mathrm{ZnO}$ polymorphs inspired by enumeration of their characteristic underlying nets. Evaluating the bulk and nanofilm stabilities of these structures with $a b$ initio calculations allows for an unprecedented overview of (nano)polymorphism in wurtzite materials. We find a rich low energy nanofilm polymorphism with a totally distinct stability ordering to that in the bulk. From this general basis we provide new insights into structural transitions observed during epitaxial growth and predictions for nanofilm stability with varying strain or thickness.
\end{abstract}

Size reduction of inorganic materials to the nanoscale often induces alternative atomic ordering, or polymorphism, relative to the most stable bulk crystal [1]. Nanoscale thin films are attractive well-defined systems to study this effect for theory and experiment. The (nano) technologically [2] important wide band gap wurtzite (wz) semiconductor $\mathrm{ZnO}$, for example, exhibits polymorphism in [0001]-oriented nanofilms on $\mathrm{Ag}(111)$ [3] and $\mathrm{Pd}(111)$ [4]. For $\leq 3$ monolayer (ML) thickness, these supported nanofilms have [0001]-stacked graphitelike flat hexagonal layers with the stacking order of hexagonal BN. This layered form of $\mathrm{ZnO}$ (layered- $\mathrm{ZnO}$ ) was first predicted to be more stable than wz-ZnO in density functional (DF) calculations for free-standing nanofilms $\leq 9$ MLs [5]. The structure of layered-ZnO appears to be driven by the elimination of the energetic cost of the electric dipole due to ionic ordering in the stacking direction in nonreconstructed [0001]-oriented wz-ZnO films. Recent theoretical work has highlighted epitaxial strain as a possible means to stabilize supported layered-ZnO nanofilms for $>9$ MLs [6]. Other DF studies of unsupported nanofilms have showed that the $\mathrm{BCT}-\mathrm{ZnO}$ [7] structure is more stable than layered- $\mathrm{ZnO}$ and nonreconstructed wz- $\mathrm{ZnO}$ for [0001]-stacked nanofilms for 4-18 MLs [8]. The lack of (0001) basal plane trigonal symmetry in BCT-ZnO, however, is incompatible with epitaxial growth on (111) surfaces of fcc metals [6] and other substrates would be required [9]. Although BCT-ZnO is not a likely competing phase in experiments where layered- $\mathrm{ZnO}$ has thus far been observed, its relevance as a structural modification is highlighted by its observation at reconstructed $\mathrm{ZnO}$ (101-0) surfaces [10] and, as a predicted phase in strained wz-ZnO nanorods [11]. Considerably expanding the handful of previously considered nanofilm polymorphs, we report over $20 \mathrm{ZnO}$ polymorphs in both nanofilm and bulk form providing us with an unprecedented overview of the (nano) structural and energetic possibilities of this important material. We show that (i) the stability range of nanofilms and their energetic ordering are radically different than that of bulk polymorphs, (ii) there exist at least three nanofilm structures with trigonal basal plane symmetry compatible epitaxial growth on fcc metal (111) surfaces that are more stable than layered- $\mathrm{ZnO}$, (iii) $\mathrm{BCT}-\mathrm{ZnO}$ is one of many structurally related and near energetically degenerate polytypic phases, (iv) with increasing thickness atomically reconstructed $\mathrm{wz}-\mathrm{ZnO}$ becomes more stable than $\mathrm{BCT}-\mathrm{ZnO}$ for $\sim 14 \mathrm{MLs}$, and is always more stable than nonreconstructed wz-ZnO, and (v) BCT-ZnO and layered-ZnO nanofilms are unstable to novel polymorphs under in-plane strain.

For all nanofilm and bulk structures, all unit cell parameters were optimized with all atoms optimized (forces $<0.01 \mathrm{eV} / \AA^{-1}$ ) with periodic DF calculations employing the PW91 functional [12], using the VASP code [13]. The projector augmented wave approach $[14,15]$ was used to describe the effect of core electrons on valence states, with the latter represented by a plane wave basis with a $500 \mathrm{eV}$ cutoff. Nanofilms were separated by over $10 \AA$ in the $c$-stacking direction to avoid spurious periodic interactions. Reciprocal space $k$-point sampling was achieved through appropriate Monkhorst-Pack grids [16]. All nanofilms have between 32 and 128 atoms per cell, with supercell tests (up to $3 \times 3$ ) showing that all energies were converged to $<0.0005 \mathrm{eV} / \mathrm{ZnO}$. We generate 24 polymorphic structures [17] using a bottom-up methodology based on nets first used to enumerate and classify bulk silicates having structures based on perpendicular linkages between two-dimensional hexagonal sheets [18]. Following the connectivity of the ten such nets in Ref. [17] with Zn-O bonding, we could generate nine distinct relaxed bulk $\mathrm{ZnO}$ polymorphs (including layered- $\mathrm{ZnO}$, wz- $\mathrm{ZnO}$, and BCT-ZnO). Such polymorphs have been predicted to be relatively stable bulk phases for wurzite materials [19] and silicon [20]. For our remaining 15 polymorphs, we created new nets based on mixing together the "primary" nets which generated the nine stablized polymorphs. New mixed nets were biased to those containing primary nets 
which previously generated more stable polymorphs. Using this method to search for stable nanofilm polymorphs has specific considerations: (i) a net which generates one bulk polymorph can often generate two [0001]-oriented nanofilms depending on the bulk cut, (ii) energetically detrimental polar surfaces can be avoided by consideration of the connectivity of the generating net.

We first focus on $4 \mathrm{ML} \mathrm{ZnO}$ nanofilms, where experiments have not yet discerned a definite preferred atomic structure. Although nonreconstructed $\mathrm{wz}-\mathrm{ZnO}$ is structurally unstable at this thickness (spontaneously converting into layered- $\mathrm{ZnO}$ ) all other polymorphs either preserved their structure or converged to structures other than layered-ZnO. Unlike unreconstructed $\mathrm{wz}-\mathrm{ZnO}$, all other considered polymorphs have no dipole in the [0001] stacking direction. In a fully ionic picture the dipole in $\mathrm{wz}-\mathrm{ZnO}$ can be quenched by atomically reconstructing the polar surfaces such that $25 \%$ of the charge on both terminating surfaces is compensated [21]. Structural relaxation and/or internal charge transfer may also assist dipole reduction [22] as in DF calculations of nonreconstructed wz-ZnO nanofilms [5,23]. Attempting to stabilize the wZ-ZnO structure in $4 \mathrm{ML}$ nanofilms, we transferred 25\% of the $\mathrm{Zn}(\mathrm{O})$ ions from the (0001)- $\mathrm{Zn}(\mathrm{O})$ surface to the (0001)-O(Zn) surface, both as separated ions, and following experimental reconstruction patterns for bulk surfaces [24,25] In all cases atomically reconstructed wz-ZnO $4 \mathrm{ML}$ nanofilms were found to be $>0.08 \mathrm{eV} / \mathrm{ZnO}$ less stable than other $4 \mathrm{ML}$ nanofilms considered.

In Fig. 1(a) we compare the energetic stabilites of $4 \mathrm{ML}$ nanofilms and the corresponding bulk phase for a wide range of polymorphic structures, relative to the most stable nanofilm or bulk phase, respectively. For any single polymorph the nanofilm stability is typically lower than in the bulk by $\sim 0.1 \mathrm{eV} / \mathrm{ZnO}$. We highlight two important general features: (i) the energy scale spanned by 4 ML nanofilms $(0.04 \mathrm{eV} / \mathrm{ZnO})$ is over four times smaller than that of the corresponding bulk structures $(0.17 \mathrm{eV} / \mathrm{ZnO})$, (ii) a significant stability reordering of corresponding structures occurs between 4 ML nanofilms and the bulk. The former suggests that interchanges between different polymorphs should be easier in nanofilms than in the bulk. On the $\mathrm{ZnO}$ (0001) surface, competition between nearly energetically degenerate reconstructed structures is found to be strongly dependent on temperature and the oxygen environment [26]. Our stoichiometric 0K structures provide a good starting point to explore such effects in nanofilms. The latter feature implied by Fig. 1(a) is that thin nanofilms are more likely to exhibit non-wz-ZnO phases. The most dramatic bulk-to-nanofilm energetic reordering occurs in the wz-to-BCT stability crossover. Using these two polymorphs as indicators of bulk and nanofilm stability, respectively, we can predict whether the energetic reordering in $\mathrm{ZnO}$ is general to other materials. The wz-to-BCT stability crossover can be quantified via the bulk-to-4ML-nanofilm destabilisation energy difference $\left(\Delta E_{\text {bulk } \rightarrow \text { nano }}\right.$, i.e., $E_{\text {nanofilm }}-E_{\text {bulk }}$, in eV/ZnO), being considerably lower for BCT-ZnO than for wz-ZnO. In Fig. 1(b) we plot the
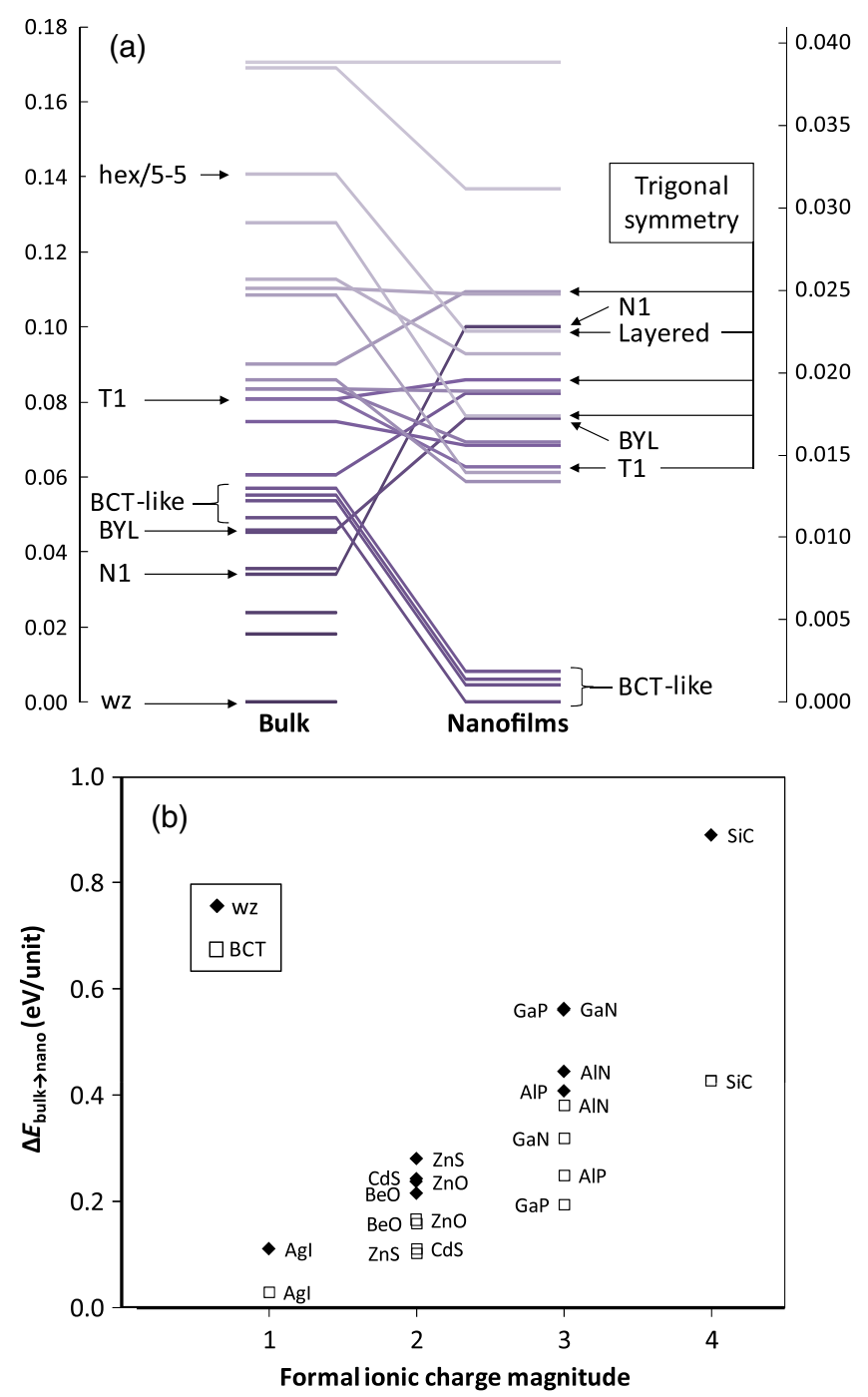

FIG. 1 (color online). (a) Comparison of relative energies $(\mathrm{eV} / \mathrm{ZnO})$ of $\mathrm{ZnO}$ polymorphs as bulk and $4 \mathrm{ML}$ nanofilms. Right and left energy $y$ axes relate to bulk and nanofilm energies, respectively. (b) $\Delta E_{\text {bulk } \rightarrow \text { nano }}(\mathrm{eV} /$ unit) for $\mathrm{wz}$ and BCT for ten wurtzite materials.

$\Delta E_{\text {bulk } \rightarrow \text { nano }}$ for BCT and wz for ten materials showing that $\mathrm{BCT}$ is always less destabilized than wz such that a bulkto-nanofilm stability crossover always occurs. The extent of the crossover is material specific, with $\Delta E_{\text {bulk } \rightarrow \text { nano }}$ linearly increasing relative to a material's formal ionic charge [with $\Delta E_{\text {bulk } \rightarrow \text { nano }}(\mathrm{wz})>\Delta E_{\text {bulk } \rightarrow \text { nano }}(\mathrm{BCT})$ for all materials]. This strongly indicates that our findings for $\mathrm{ZnO}$ are applicable to many other materials. In the remainder we explore various physical implications suggested by the unique overview provided by Fig. 1(a).

Consistent with previous studies the most energetically stable $4 \mathrm{ML}$ nanofilm has the BCT-ZnO structure. Within a very small energy range above BCT-ZnO for both bulk phases $(0.01 \mathrm{eV} / \mathrm{ZnO})$ and nanofilms $(0.0025 \mathrm{eV} / \mathrm{ZnO})$, we find three other structures. We call these structures BCT-like as they can be regarded as polytypes (i.e., different stacking sequences of structurally similar infinite 
two-dimensional layers [27]) with the constituent layers being parallel to the $c$ axis. Polytypism also occurs in the stacking of the $a-b$ aligned hexagonal layers with wurtzite (aligned stacking) and zinc blende (antialigned stacking) being the end members. For BCT-like structures, $\mathrm{BCT}$ is one end member with aligned layer stacking, with an antialigned layer stacked structure (which we name BCT- $\omega$, see Fig. 2) forming the other end member. Stacking variations allow potentially infinite possibilities, each giving rise to a distinct BCT-like polytype. Although close in energy, no structural changes in BCT- $\omega$ occurred in classical NPT molecular dynamics calculations (1 atm and $300-500 \mathrm{~K}$ for $100 \mathrm{ps}$ ), using dedicated potentials [28] and the GULP code [29]. The dynamically stability of BCTlike polytypes (and $c$-stacked polytypes) may be due to the energetically expensive requirement of wholesale changes in bonding between all aligned layers for interconversions.

Although having high stabilities, due to their lack of trigonal (0001) basal plane symmetry, the BCT-like $\mathrm{ZnO}$ nanofilms are unlikely to explain the experimental observation that the fcc metal-supported layered- $\mathrm{ZnO}$ phase disappears for $\geq 3$ ML [3,4]. Unlike the observed of $\mathrm{Zn}$ deficiency on bulk-terminated polar (0001)-Zn surfaces [25], in Ref. [3], the 1:1 ZnO stoichiometry was kept fixed within all layers indicating that any dipole reduction is via another mechanism. The good structural agreement between experiment and theoretical calculations for freestanding layered- $\mathrm{ZnO}$ nanofilms [5] for the very thin 1-3 ML regime, indicates that metal support has a minor effect on nanofilm structure. Calculations on 1 ML layered$\mathrm{ZnO}$ supported on $\mathrm{Ag}(111)$ [30] also show no indication of metal-ZnO charge transfer, confirming the rather passive role of the Ag support. The experimentally prepared
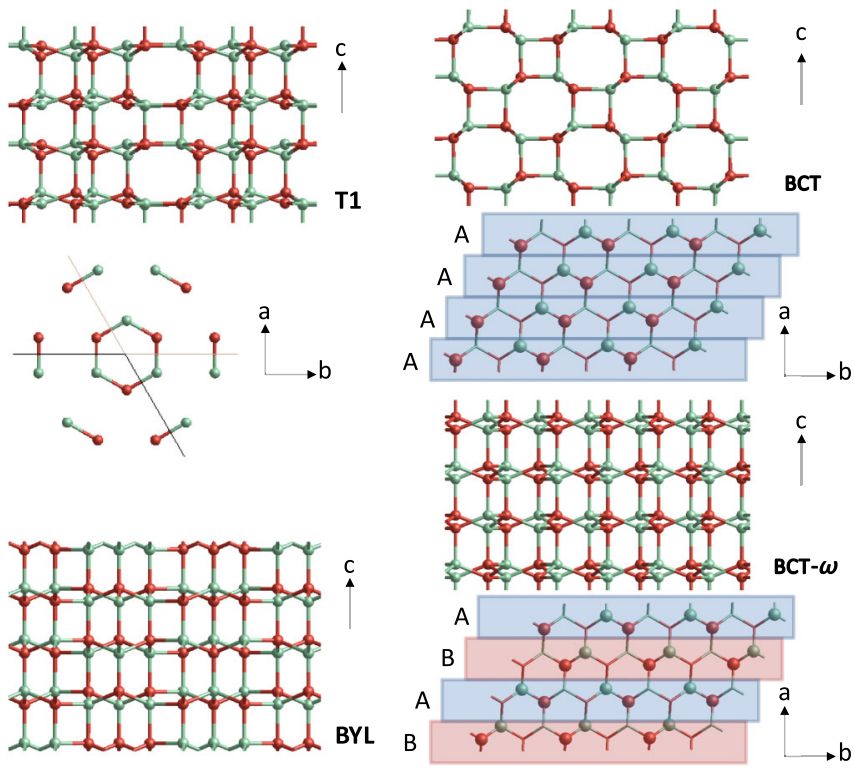

FIG. 2 (color online). Selected polymorphs. Top left: T1 with its trigonal basal plane, bottom left: BYL, right: BCT (upper) and BCT- $\omega$ (lower) with respective $c$-aligned stacking sequences.
3.5 ML $\mathrm{ZnO} \mathrm{Ag}(111)$-supported nanofilm has a $55 \%$ $4 \mathrm{ML}$ coverage (the third ML coverage being $5 \%$ under complete), and thus is formed by $50 \%$ of stoichiometric $4 \mathrm{ML} \mathrm{ZnO}$.

Considering our set of stoichiometric nonpolar $4 \mathrm{ML}$ nanofilms, we find three which have trigonal (0001) basal plane symmetry and are more energetically stable than layered- $\mathrm{ZnO}$. The most stable of these (T1) is labeled in Fig. 1 (see also Fig. 2). In Table I we compare the calculated structures of $\mathrm{T} 1-\mathrm{ZnO}$ and layered-ZnO $4 \mathrm{ML}$ nanofilms and bulk $\mathrm{wz}-\mathrm{ZnO}$ with the experimental interlayer structural data for the 3.5 and 4.5 ML samples from Ref. [2]. For interatomic distances between layers 1 and 2 in both 3.5 and 4.5 ML experimental samples, the best match with the calculated data is mainly for $4 \mathrm{ML}$ layered- $\mathrm{ZnO}$.

Following Ref. [3] this strongly indicates that this polymorph dominates the first two MLs. For layers 3 and 4 the interatomic $\mathrm{Zn}-\mathrm{O}$ and $\mathrm{Zn}-\mathrm{Zn}$ distances strongly point to an alternative structure in both samples, with the calculated bulk wz-ZnO interlayer distances matching well with experiment. Although free-standing $4 \mathrm{ML}$ wz-ZnO nanofilms are inherently structurally unstable, it is perhaps possible in experiment that epitaxial effects and/or charge transfer from the support may help to stabilize more bulklike $4 \mathrm{ML}$ nanofilm structures. Alternatively, the experimental data may point to another polymorphic structure for the 3.5 and/ or 4.5 ML nanofilms. In Table I, the calculated interlayer data for the $\mathrm{T} 1-\mathrm{ZnO} 4 \mathrm{ML}$ nanofilm shows good agreement with the experimental data for the interatomic distances between layers 2 and 3, particularly for the 4.5 ML sample. Allowing for the estimated experimental error $( \pm 0.1 \AA)$ an interpretation of the experimental data for layers 2 and 3 by layered- $\mathrm{ZnO}$ or $\mathrm{wz}-\mathrm{ZnO}$ may also be possible. However, that there is at least one other candidate nanofilm structure that is fully compatible with the experimental interlayer data opens the possibility that such polymorphs could play a role in the observed structural instability of metal-supported $\mathrm{ZnO}$ for $>2 \mathrm{ML}[3,4]$.

TABLE I. Comparison of differences in calculated structural data [interlayer $\mathrm{Zn}-\mathrm{Zn}\left(d_{\mathrm{Zn}-\mathrm{Zn}}\right)$ and $\mathrm{Zn}-\mathrm{O}\left(d_{\mathrm{Zn}-\mathrm{O}}^{\prime}\right)$ distances] for layered$\mathrm{ZnO}$ and $\mathrm{T} 1-\mathrm{ZnO} 4 \mathrm{ML}$ nanofilms and bulk wz-ZnO with experimental data for 3.5 and 4.5 ML [3]. Best matches are underlined.

\begin{tabular}{|c|c|c|c|c|c|c|}
\hline \multirow[b]{2}{*}{$d_{\mathrm{Zn}-\mathrm{Zn}}(\AA)$} & \multicolumn{3}{|c|}{$3.5 \mathrm{ML}$} & \multicolumn{3}{|c|}{$4.5 \mathrm{ML}$} \\
\hline & $\begin{array}{c}\text { Layer } \\
1-2\end{array}$ & $\begin{array}{c}\text { Layer } \\
2-3\end{array}$ & $\begin{array}{c}\text { Layer } \\
3-4\end{array}$ & $\begin{array}{c}\text { Layer } \\
1-2\end{array}$ & $\begin{array}{c}\text { Layer } \\
2-3\end{array}$ & $\begin{array}{c}\text { Layer } \\
3-4\end{array}$ \\
\hline Exp. & 2.30 & 2.53 & 2.66 & 2.25 & 2.54 & 2.62 \\
\hline Layered & $\underline{0.00}$ & -0.15 & -0.36 & $\underline{0.05}$ & -0.16 & -0.32 \\
\hline $\mathrm{T} 1$ & 0.18 & -0.05 & -0.18 & 0.23 & -0.06 & -0.14 \\
\hline $\begin{array}{l}\text { wz (bulk) } \\
d_{\mathrm{Zn}-\mathrm{O}}^{\prime}(\AA)\end{array}$ & 0.35 & 0.12 & -0.01 & 0.40 & 0.11 & -0.03 \\
\hline Exp. & 2.27 & 2.40 & & 2.10 & 2.26 & \\
\hline Layered & $\underline{0.06}$ & -0.03 & & 0.23 & 0.11 & \\
\hline $\mathrm{T} 1$ & $-\overline{0.13}$ & -0.18 & & $\underline{0.04}$ & -0.03 & \\
\hline wz (bulk) & -0.26 & -0.39 & & -0.09 & -0.25 & \\
\hline
\end{tabular}


Relative nanofilm structural stability can also be influenced by thickness. A previous study showed that BCT-ZnO is more energetically stable than layered- $\mathrm{ZnO}$ and unreconstructed wz-ZnO from 4-18 ML [8]. From Fig. 1 we can choose a structure having an energetic stability between $\mathrm{BCT}-\mathrm{ZnO}$ and layered-ZnO for $4 \mathrm{ML}$, and between wz-ZnO and BCT-ZnO in the bulk phase (e.g., N1 or BYL [31] in Fig. 1). We can then predict that at some nanofilm thickness $>4 \mathrm{ML}$ this phase will be more energetically favored than $\mathrm{ZnO}$ BCT. In Fig. 3(a) we plot the energetic stability of BYL-ZnO (see also Fig. 2) and that of BCT-ZnO relative to nanofilm thickness. Extrapolating from the calculated data points for $8,12,16$, and $20 \mathrm{ML}$, we predict a crossover at a thickness of approximately $26 \mathrm{ML}$ at which BYL-ZnO [blue line, Fig. 3(a)] will be more stable than BCT-ZnO [gray line, Fig. 3(a)]. Following previous work $[5,6,8]$, we also include unreconstructed $\mathrm{wz}-\mathrm{ZnO}$ films (structurally stable for $>9 \mathrm{ML}$ only) for comparison [green line, Fig. 3(a)]. We find that the BCT-to-BYL crossover occurs slightly before the thickness at which it is estimated that unreconstructed $\mathrm{wz}-\mathrm{ZnO}$ becomes more stable than BCT$\mathrm{ZnO}$ ( 28 ML [8]). We also consider reconstructed wz-ZnO nanofilms. Although for $4 \mathrm{ML}$ we could not stabilize the wz$\mathrm{ZnO}$ structure, for $\geq 8 \mathrm{ML}$ nanofilms atomic reconstruction can stabilize the wz-ZnO structure. Here we employ a simple individual ion transfer approach to quench the dipole in the 8-20 ML wz-ZnO nanofilms. As expected from our attempts with $4 \mathrm{ML}$ nanofilms, $8 \mathrm{ML}$ free-standing atomically reconstructed $\mathrm{wz}-\mathrm{ZnO}$ nanofilms are considerably less stable than most other nanofilms (e.g., BCT-ZnO or $\mathrm{BYL}-\mathrm{ZnO}$ ). For increasing thickness, however, the stability of such nanofilms rapidly increases such that atomically reconstructed $\mathrm{wz}-\mathrm{ZnO}$ becomes the most energetically stable $\mathrm{ZnO}$ phase for $>16 \mathrm{ML}$ [red line, Fig. 3(a)], well before either BYL-ZnO or unreconstructed wz-ZnO nanofilms start to energetically compete with BCT-ZnO. For all thicknesses considered, and by extrapolation, for all thicknesses $\geq 9 \mathrm{ML}$, our results imply that atomically reconstructed
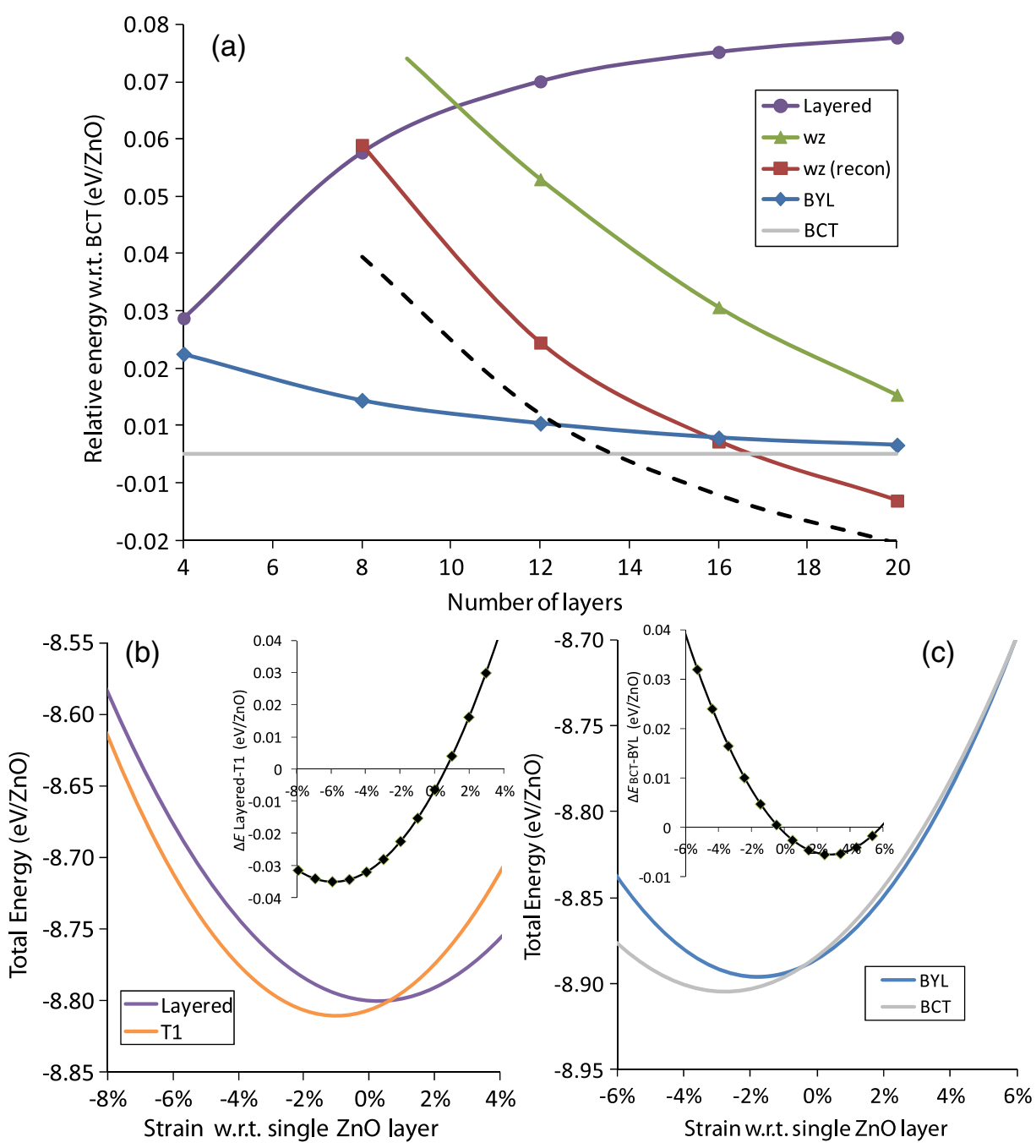

FIG. 3 (color online). (a) Selected nanofilm energetic stability variation with thickness, (b) energy variation with in-plane strain for $4 \mathrm{ML}$ layered-ZnO versus $\mathrm{T} 1-\mathrm{ZnO}$, (c) energy variation with in-plane strain for $8 \mathrm{ML} \mathrm{BCT}-\mathrm{ZnO}$ versus BYL-ZnO. Strains are relative to $1 \mathrm{ML}$ layered-ZnO. Insets show polymorph energy differences. 
wz- $\mathrm{ZnO}$ should be always more stable than unreconstructed $\mathrm{wz}-\mathrm{ZnO}$. We note that the individual ion transfer method has been estimated to be $0.3 \mathrm{eV}$ less efficient per transferred ion than more sophisticated atomic reconstructions [25]. In Fig. 3(a) a dashed line shows the estimated downward shift due to such an improved model that has the main effect of causing the crossover with BCT-ZnO to occur at $>14 \mathrm{ML}$ rather than $>16 \mathrm{ML}$.

Finally, we consider the effect of uniform in-plane $a-b$ strain on relative nanofilm polymorphic stability. We examine two systems: (i) $\mathrm{T} 1-\mathrm{ZnO}$ versus layered-ZnO in $4 \mathrm{ML}$ nanofilms, and (ii) BYL-ZnO versus BCT-ZnO in $8 \mathrm{ML}$ nanofilms. In the first case, as the two polymorphs are compatible with growth on metal (111) substrates, our calculations indirectly investigate the possible effect of epitaxy on nanofilm polymorphism. In a similar study in Ref. [5] only unreconstructed $4 \mathrm{ML}$ wz-ZnO was used as a comparison with $4 \mathrm{ML}$ layered- $\mathrm{ZnO}$ and thus the latter was proposed to be the most stable nanofilm structure for all strains. In Fig. 3(b) it can be seen that, although layered- $\mathrm{ZnO}$ is favored for larger positive strains, for small positive strains through to moderately compressive strains $\mathrm{T} 1-\mathrm{ZnO}$ is favored energetically. Our results imply that substrates with in-plane lattice mismatches that induce small compressive epitaxial strains could help to stabilize $\mathrm{T} 1-\mathrm{ZnO}$. In the second case we investigate the role of strain in potentially altering the energetic ordering of low energy free-standing BYL-ZnO $8 \mathrm{ML}$ nanofilms relative to BCT-ZnO. For $8 \mathrm{ML}$ nanofilms reconstructed $\mathrm{wZ}-\mathrm{ZnO}$ is not energetically competitive with BCT-ZnO or BYL-ZnO but the latter two phases are close in energy. Under small positive strains we find that there is a small window of excess stability for the BYL-ZnO phase where energetic stability of the two nanofilms is inverted [see Fig. 3(c)]. These results further confirm other theoretical studies which suggest that mechanical forces applied to $\mathrm{ZnO}$ nanosystems may be a fertile route to access new polymorphs [6,11].

In summary, we have developed a method to generate a wide range of new low energy nanofilm and bulk polymorphs using nets as a basis. Consideration of the energetics of these structures permits an unprecendented overview of nanoscale polymorphism in $\mathrm{ZnO}$ and its evolution with size and strain. Our results provide convincing evidence that low energy polymorphism in nanofilms of wurtzite materials should be very rich, and significantly beyond the handful of polymorphs previous considered in the literature. Our results strongly suggest that many new nanofilm polymorphs should be experimentally accessible, and in some cases (e.g., T1-ZnO), may have even already been observed.

This study has been supported by the Spanish Government (Grants No. FIS2008-02238 and No. MAT2012-30924) and by the Generalitat de Catalunya (Grants No. 2009SGR1041 and No. XRQTC). We also thank Dr. J. Goniakowski for useful discussions, and Dr. M. A. Zwijnenburg for his assistance in finding some of the structures.
*Corresponding author.

s.bromley@ub.edu

[1] A. Navrotsky, Proc. Natl. Acad. Sci. U.S.A. 101, 12096 (2004).

[2] X. Wang, J. Song, and Z. L. Wang, J. Mater. Chem. 17, 711 (2007).

[3] C. Tusche, H. L. Mayerheim, and J. Kirschner, Phys. Rev. Lett. 99, 026102 (2007).

[4] G. Weirrum, G. Barcaro, A. Fortunelli, F. Weber, R. Schennach, S. Surnev, and F. P. Netzer, J. Phys. Chem. C 114, 15432 (2010)

[5] C. L. Freeman, F. Claeyssens, N.L. Allan, and J.H. Harding, Phys. Rev. Lett. 96, 066102 (2006).

[6] D. Wu, M. G. Lagally, and F. Liu, Phys. Rev. Lett. 107, 236101 (2011).

[7] BCT refers to the International Zeolite Association code for the Body Centered Tetragonal framework which has the underlying 4-connected topology of the silicate Mg-BCTT.

[8] B. J. Morgan, Phys. Rev. B 80, 174105 (2009).

[9] B. J. Morgan, Phys. Rev. B 82, 153408 (2010).

[10] M.-R. He, R. Yu, and J. Zhu, Angew. Chem., Int. Ed. 51, 7744 (2012).

[11] J. Wang, A. J. Kulkarni, M. Zhou, K. Sarasamak, and S. Limpijumnong, Phys. Rev. Lett. 97, 105502 (2006).

[12] J. P. Perdew and Y. Wang, Phys. Rev. B 45, 13244 (1992).

[13] G. Kresse and J. Hafner, Phys. Rev. B 47, 558 (1993).

[14] P. E. Blöchl, Phys. Rev. B 50, 17953 (1994).

[15] G. Kresse and D. Joubert, Phys. Rev. B 59, 1758 (1999).

[16] H. J. Monkhorst and J. D. Pack, Phys. Rev. B 13, 5188 (1976).

[17] See Supplemental Material at http://link.aps.org/ supplemental/10.1103/PhysRevLett.110.245501 for the optimized cell parameters, fractional coordinates, and energetic stabilities of all 24 hexagonal sheet based bulk $\mathrm{ZnO}$ polymorphs.

[18] J. V. Smith, Am. Mineral. 62, 703 (1977).

[19] M. A. Zwijnenburg, F. Illas, and S. T. Bromley, Phys. Rev. Lett. 104, 175503 (2010).

[20] M. A. Zwijnenburg, K. E. Jelfs, and S. T. Bromley, Phys. Chem. Chem. Phys. 12, 8505 (2010).

[21] P. W. Tasker, J. Phys. C 12, 4977 (1979).

[22] J. Goniakowski, F. Finocchi, and C. Noguera, Rep. Prog. Phys. 71, 016501 (2008).

[23] A. Wander and N. M. Harrison, J. Chem. Phys. 115, 2312 (2001).

[24] J. V. Lauristen, S. Porrsgaard, M. K. Rasmussen, M. C. R. Jensen, R. Bechstein, K. Meinander, B.S. Clausen, S. Helveg, R. Wahl, G. Kresse, and F. Besenbacher, ACS Nano 5, 5987 (2011).

[25] O. Dulub, U. Diebold, and G. Kresse, Phys. Rev. Lett. 90, 016102 (2003).

[26] M. Valtiner, M. Todorova, G. Grundmeier, and J. Neugebauer, Phys. Rev. Lett. 103, 065502 (2009).

[27] G. S. Oleinik and N. V. Danilenko, Russ. Chem. Rev. 66, 553 (1997).

[28] L. Whitmore, A. A. Sokol, and C. R. A. Catlow, Surf. Sci. 498, 135 (2002).

[29] J. D. Gale, Z. Kristallogr. 220, 552 (2005).

[30] I. Demirolgu, D. Stradi, F. Illas, and S. T. Bromley, J. Phys. Condens. Matter 23, 334215 (2011).

[31] The BYL-ZnO polymorph has the same underlying net as the mineral beryllonite: http://rcsr.anu.edu/nets/byl. 